\title{
Building and Operating an E-business Platform - Technical Challenges and Other Pitfalls
}

\author{
Peter Zoller \\ BMW AG \\ Sapporobogen 6-8 \\ 80788 München \\ peter.zoller@bmw.de
}

\begin{abstract}
This short paper describes the challenges faced and experiences gathered during build-up and first year of operation of the BMW Group b2bplatform. It is divided into three sections: first a brief outline on the project and the main difficulties during system implementation is given. Afterwards challenges and experiences concerning operation of the platform are presented. The paper ends with conclusions.
\end{abstract}

\section{Project Details and Difficulties During Implementation}

In December 2000 the BMW management board decided to implement an e-businessplatform in order to meet the future challenges of electronic business. After evaluating tools and products for the central services and components, in June 2002 the build-up of the b2b-platform started.

The core components of the platform are a portal frontend providing role-based navigation, an authentication and authorization service (EAM, Enterprise Access Management), an LDAP-based user and role store (LAAS) and a web based interface for delegated user management. The designated user group comprised about 8000 partner users from roughly 2000 companies, mainly located in Europe. The rollout of the B2B-Portal was planned for the beginning of 2003.

Although using a standard web architecture and limiting both portal functionality and supported client systems in order to minimize technical risks, several technical challenges had to be faced:

- The EAM had an LDAP interface and could smoothly be integrated with LAAS, but performance of the whole system was bad in the beginning because of different optimization strategies of the two components.

- The configuration of the web servers containing the EAM plugin proved to be error-prone and extremely sensitive against changes of the server configuration (even against changes in the operating system)

\section{System Operation - Good Performance and High Availability Are Not Enough}

Since June 2003 the BMW b2b-platform (partner portal) is available under https://b2b.bmw.com. On workdays we currently have about 2000 user sessions (logins), the average login in the central components takes about $350 \mathrm{~ms}$ and building 
up the personalized portal page takes about 2-3 seconds for the initial access. Availability within the first 6 months has exceeded $99.8 \%$, the system runs very stable. Meanwhile about 12.000 external and more than 17.000 internal users have access to the b2b-platform.

Nevertheless we have been confronted with some serious problems:

- Browser problems: even though we have restricted the set of supported browsers and avoided the use of applets and Java script as far as possible, browser problems caused the majority of helpdesk calls. The reasons were either real browser bugs, misconfigurations on client side (JDK, cookies, caching) or seldom html/java script coding errors in the applications.

- Time-consuming and expensive user support: end user error reports are often unexact ("portal doesn't work", "portal is slow") and do not distinguish between browser, internet-connection, portal and applications. Since the platform is a rather complex system and one important component (the user's browser) is not within our reach, support and debugging is very difficult and time consuming.

- Integrating applications into a central platform is a complex process. A variety of departments is involved and since the number of applications grew rapidly (currently more than 35 have already been integrated), additional resources and improved integration-processes were needed.

- Configuration management is very costly: a valid system configuration consists of numerous configuration files and database/LDAP entries. Migrating a new configuration (e.g. after integrating a new application) from test environment to integration environment and afterwards to production environment requires a lot of manual configuration since many commercial products don't support configuration propagation out of the box. Some vendors even don't offer any interfaces at all. Some technical features of the platform are not used because efficient maintenance would not be possible.

\section{Conclusions}

The launch of the new BMW partner portal in June 2003 has been very successful, especially considering the tight time schedule of the project. The key to success was the use of standards and the strong restriction of functionality that was implemented. Although availability and performance of the system are very good, operating the platform provided the major challenges of this project:

- End user support is costly especially if the audience is as heterogeneous as typically in b2b-platforms. The reasons are technically insufficient error reports on the one hand and an infrastructure that is very complex to debug on the other hand.

- Configuration Management of complex infrastructures is not supported sufficiently by tools and standards.

- Developing efficient processes for the technical integration of applications into the platform considering organizational structures is an essential task.

- The use of standards usually guarantees a general technical compatibility of components but leaves enough room for individual usage and optimization strategies. This might result in an absolutely unacceptable performance of the platform, although the standard is obeyed and the interfaces are optimized by each component. 